\title{
Fiber optics and basic physics
}

\author{
Ajoy Ghatak
}

Ajoy Ghatak, "Fiber optics and basic physics," Proc. SPIE 9663, Eighth International Topical Meeting on Education and Training in Optics and Photonics, 96632H (6 October 2003); doi: 10.1117/12.2208384

SPIE Event: Eighth International Topical Meeting on Education and Training in SPIE. Optics and Photonics, 2003, Tucson, Arizona, United States 


\title{
Fiber optics and basic physics
}

\author{
Ajoy Ghatak \\ Physics Department, Indian Institute of Technology, Delhi, Hauz Khas, New Delhi, India 110016 \\ telephone: +911126591363; fax:+911126865039; e-mail: ajoykghatak@yahoo.com
}

\begin{abstract}
Study of fiber optics allows us easy visualization of many physical concepts like normal modes and super modes, polarization dependent phenomena, nonlinear phenomena like solitons etc. Indeed fiber optics also allows us easy elucidation of quantum mechanical phenomena like quantization and tunneling.

(C)2003 Optical Society of America
\end{abstract}

OCIS codes: (060.2310) Fiber optics

The field of fiber optics has seen an explosive growth over the last three decades because of its numerous applications in many diverse areas like communications, sensors, signal processing etc. As such, it has become very necessary to teach such a course as a part of the undergraduate program in Electrical Engineering as well as in Physics. In addition to its many facet applications, a study of this field enables easy visualization of various physical concepts like normal modes and super modes, dispersion and chirping, nonlinear phenomena like solitons, polarization dependent phenomena, etc. Such a course also affords easy elucidation of quantum mechanical phenomena like quantization and tunneling. The talk will discuss several aspects of this exciting field and how it allows easy understanding of difficult physical concepts. 